\title{
Vasospastic Angina and Role of Cardiac Catheterization
}

\author{
Robert Kleyman ${ }^{1}$, Rajiv Goyal ${ }^{1}$, Neha Patel $^{1}$, Jessica Joseph ${ }^{2}$, Rami Akel ${ }^{2}$ \\ 1. Internal Medicine, Graduate Medical Education, Regional Medical Center Bayonet Point, Hudson, USA 2. Cardiology, \\ Graduate Medical Education, Regional Medical Center Bayonet Point, Hudson, USA
}

Corresponding author: Robert Kleyman, robert.kleyman@hcahealthcare.com

\begin{abstract}
Prinzmetal angina, also known as vasospastic or variant angina, is defined as an intermittent focal coronary artery spasm often associated with an atherosclerotic lesion near the site of spasm. It is caused by a focal or diffuse spasm of the smooth layer of the arterial wall of an epicardial coronary artery. Acute infarctions or malignant arrhythmias may develop during spasm-induced ischemia. Evaluation includes observation of echocardiogram (EKG) for transient ST elevations during discomfort; diagnosis is confirmed with coronary angiography using provocative testing. We describe two cases of patients who presented for non-cardiac complaints, but had episodes of vasospastic angina during their hospitalization. Both underwent cardiac catheterization with differing results, demonstrating the importance of catheterization in patients who experience vasospastic angina.
\end{abstract}

Categories: Cardiology

Keywords: prinzmetal, angina, st-elevation, coronary occlusion, transient ischemia, catheterization, percutaneous intervention, ventricular arrhythmias

\section{Introduction}

Vasospastic angina is an intermittent focal coronary artery spasm that is defined as having a transient or sustained reduction in the diameter by more than $50 \%$ in an arterial segment with insignificant $(<25 \%)$ baseline narrowing [1]. It is caused by a focal or diffuse spasm of the smooth muscle layer of the arterial wall of an epicardial coronary artery [2,3]. Vascular smooth muscle hyper-reactivity is thought to be central to the pathogenesis of vasospastic angina [2,3]. The prevalence of vasospastic angina is higher in individuals from Japan compared to the Caucasian population [4]. Multiple vasoconstrictors have been used to provoke coronary spasm including acetylcholine, serotonin, histamine, noradrenaline, and dopamine, suggesting that a single receptor pathway cannot explain the phenomenon. Chest discomfort is similar to angina pain, described as being more severe and occurring typically at rest, most frequently during the hours of 12 a.m. to early morning with associated transient ST segment elevation. Each episode lasts on average from five to 15 minutes, but can be longer. There are many causes of vasospastic angina some of which include use of tobacco and recreational drugs including cocaine and amphetamine, guide wire balloon dilatation at the time of percutaneous coronary intervention (PCI), and chronic obstructive pulmonary disease (COPD) to name a few. An underlying atherosclerotic coronary artery disease $(\mathrm{CAD})$ can play a role in vasospastic angina events. A recent cohort study reported that approximately $10 \%$ of patients with underlying atherosclerotic CAD presented with sudden cardiac death related to vasospastic angina events [5]. Diagnosis of vasospastic angina can be made using the Coronary Vasomotion Disorders International Study Group (COVADIS) guidelines, which includes nitrate-responsiveness, transient ischemic echocardiogram (EKG) changes, and coronary artery spasms [6].

\section{Case Presentation}

\section{Case 1}

A 59-year-old Caucasian male with no history of heart disease presented with shortness of breath with reported medical history of chronic obstructive pulmonary disease and tobacco use. He was treated for a COPD exacerbation without complications. On day 6 of his hospitalization, at approximately 12 a.m., he developed acute onset substernal, non-radiating chest pain with associated nausea. He denied previous history of similar symptomatology. At the time of event, he was hypertensive with a blood pressure of $190 / 110 \mathrm{mmHg}$, tachycardic at a rate of 150 beats per minute, tachypnic with a respiratory rate of 24 breaths per minute, saturating at $97 \%$ on three liters nasal cannula. He was actively being monitored on telemetry. At the time of symptom onset, he was found to have Torsades de pointes (figure 1) with progression to atrial fibrillation. 


\section{Cureus}

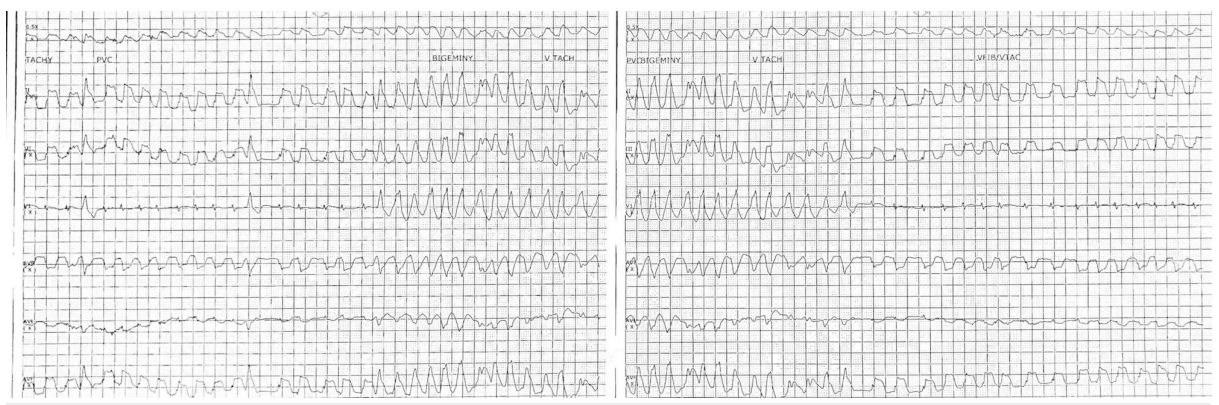

FIGURE 1: Telemetry strip demonstrating nonsustained ventricular tachycardia

EKG obtained during the event demonstrated new onset atrial fibrillation with rapid ventricular response and significant ST elevations in the anterolateral leads that was significantly different when compared to admission EKG (figures 2-3).

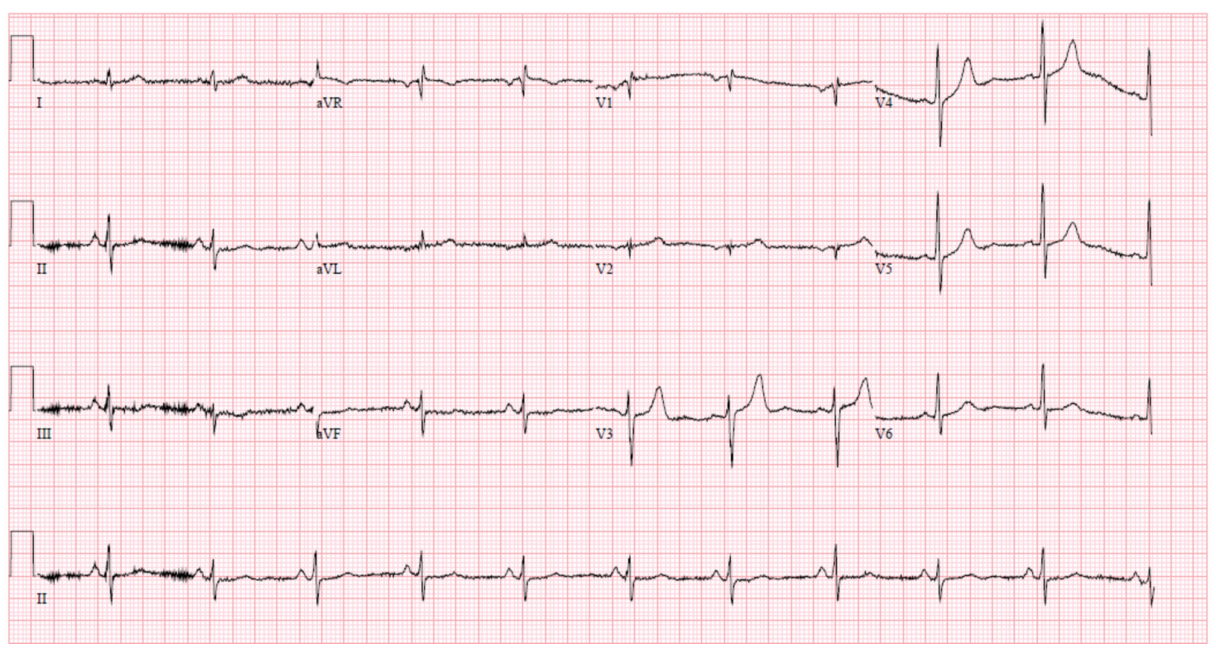

FIGURE 2: Baseline 12-lead electrocardiogram from admission

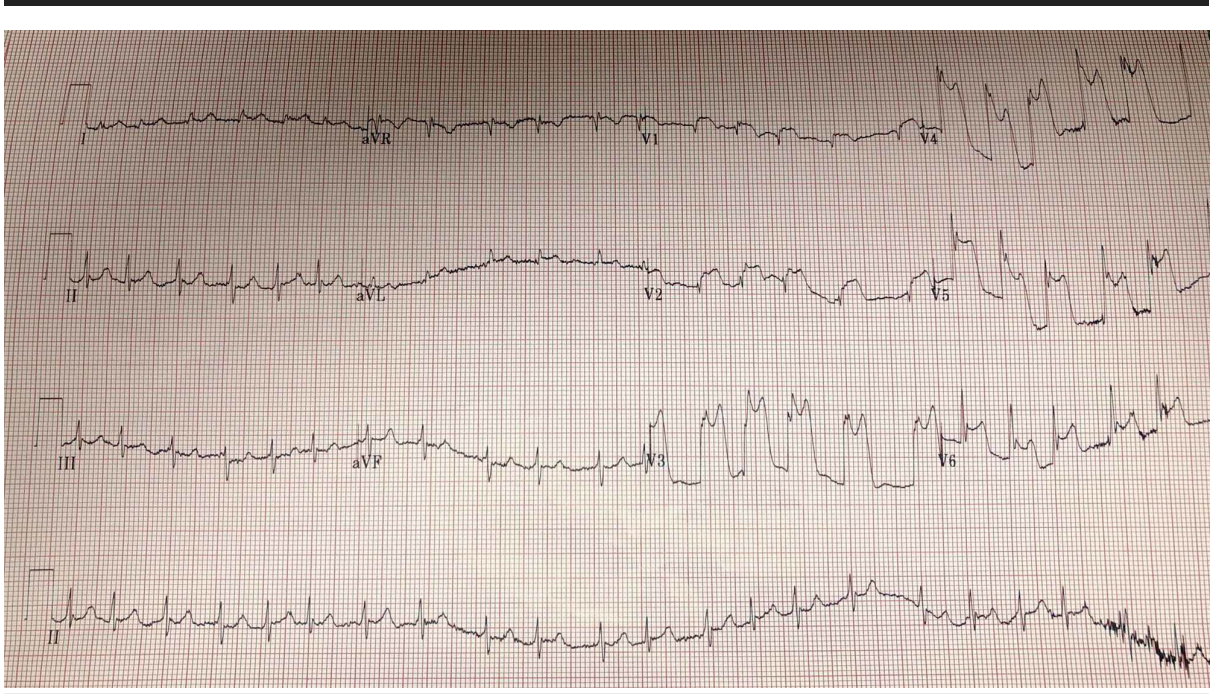

FIGURE 3: Atrial fibrillation with rapid ventricular response and significant ST-elevations in anteriolateral leads (V1-V6)

He was subsequently started on a heparin and nitroglycerin drip, along with an amiodarone drip and 


\section{Cureus}

transferred to the intensive care unit for close hemodynamic monitoring. His symptoms resolved shortly after. A repeat EKG revealed resolution of ST elevations (figure 4).

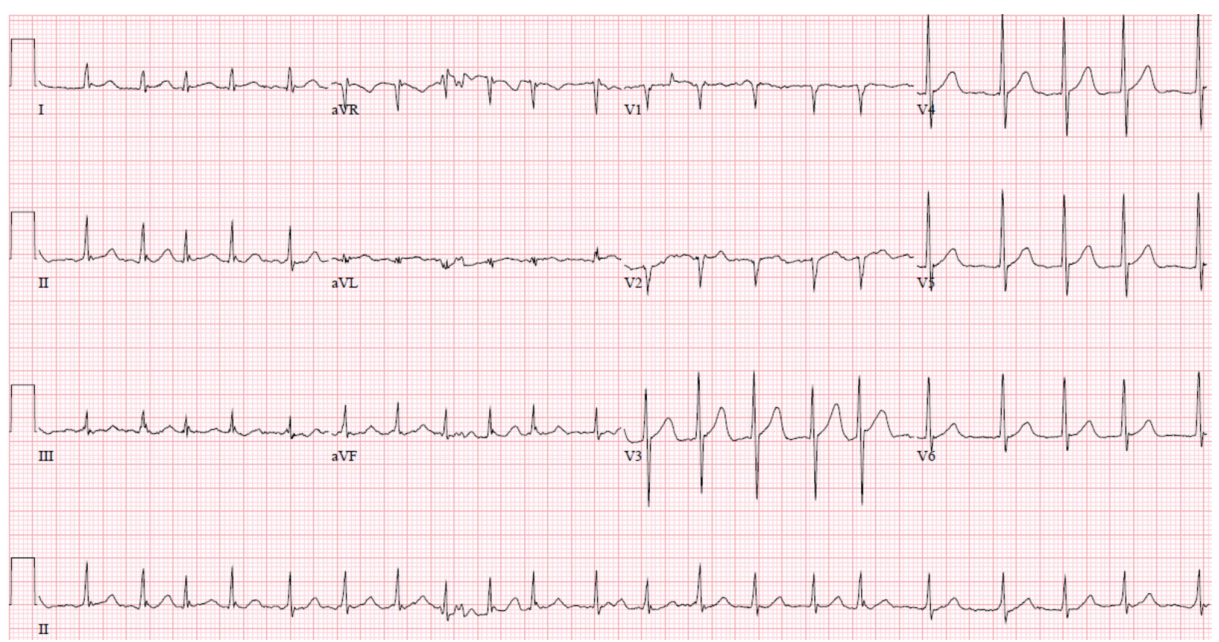

FIGURE 4: 12-lead echocardiogram post nitroglycerin administration demonstrating resolution of ST-elevations

\section{Case 2}

A 70-year-old Caucasian male presented with lightheadedness and dizziness. He reported a past medical history of sub-massive pulmonary embolism requiring thrombolysis on anticoagulation, type 2 diabetes mellitus, recent bleeding duodenal ulcers status post argon plasma coagulation (APC) with clipping and active tobacco use. In the preceding months, he admitted to recurrent epigastric abdominal complaints described as a burning sensation that he attributed to chronic gastroesophageal reflux disease (GERD). On admission, he was found to have a hemoglobin of $4.3 \mathrm{~g} / \mathrm{dL}$ and was treated for acute blood loss anemia secondary to a gastrointestinal (GI) bleed. During his hospitalization, he developed non-radiating epigastric/substernal chest pain described as a burning sensation. EKG obtained during the event revealed sinus rhythm with new ST elevations in inferolateral leads when compared to EKG obtained at the time of admission (figures 5-6).

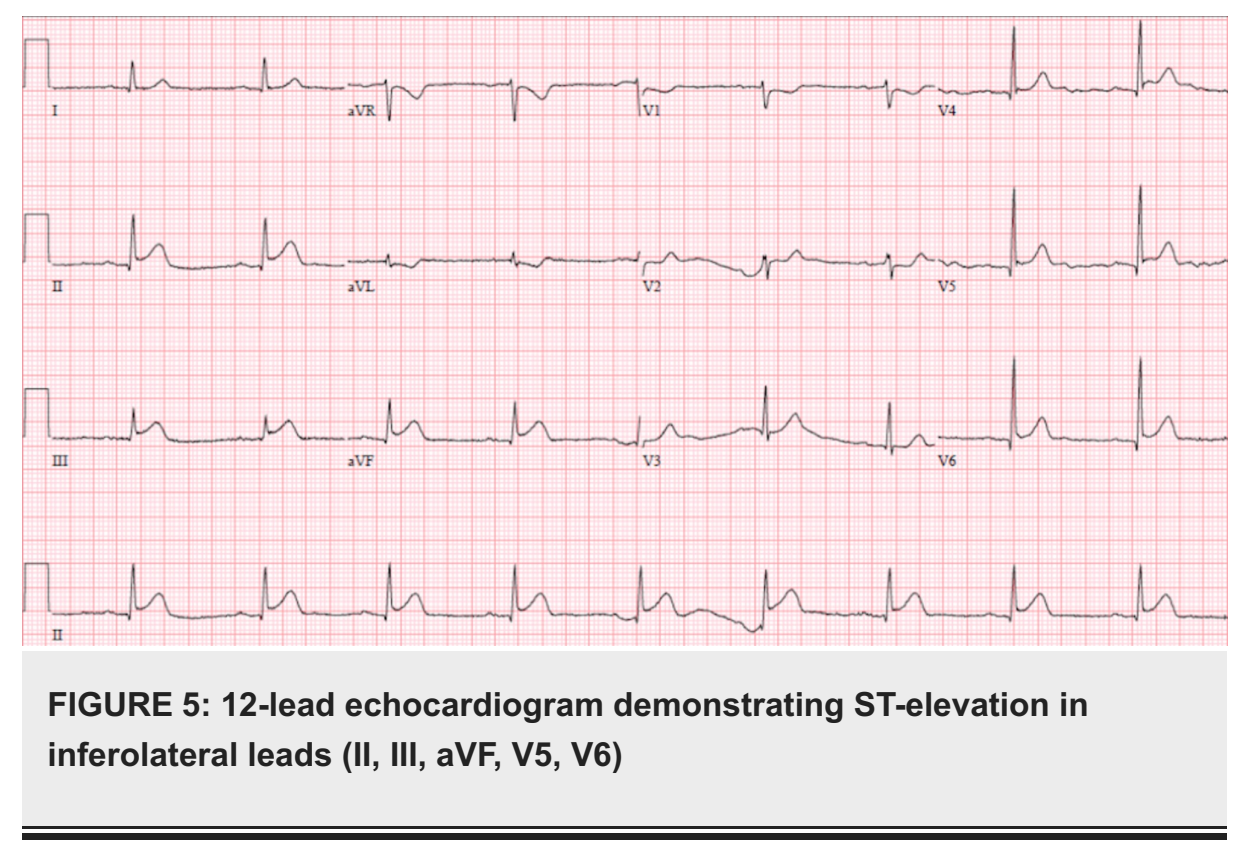




\section{Cureus}

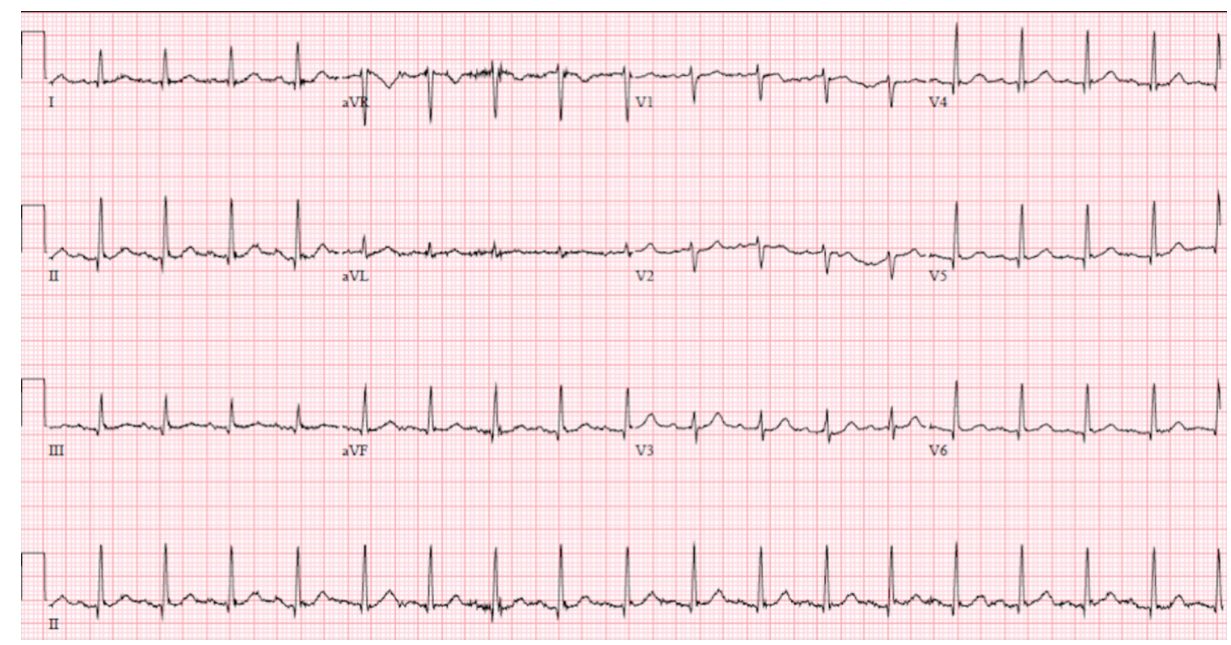

FIGURE 6: Baseline 12-lead echocardiogram from admission

EKG findings were thought to be consistent with vasospastic angina and patient was subsequently started on isosorbide mononitrate and amlodipine. However, acute coronary syndrome could not be ruled out at the time and patient received full dose aspirin, $325 \mathrm{mg}$, and atorvastatin $80 \mathrm{mg}$. Due to GI bleed on admission, he was unable to be placed on a heparin drip. Patient's symptoms subsided after administration of nitrate. Repeat EKG revealed resolution of ST elevations (figure 7). Troponins were obtained and peaked at 7.49 $\mathrm{ng} / \mathrm{mL}$. Patient was closely monitored for the remainder of the night and was taken for left heart catheterization in the morning.

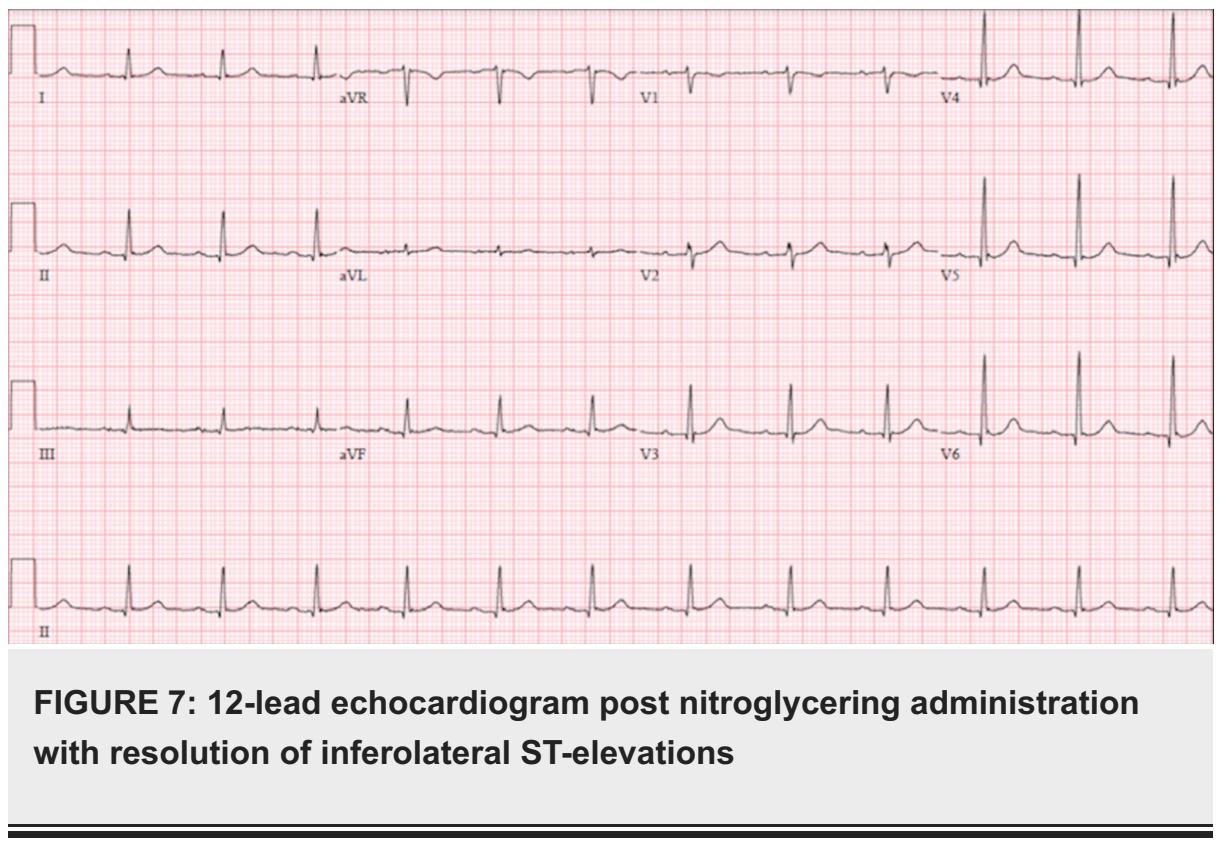

\section{Outcome}

In each of the cases, patients were taken for cardiac catheterization. Patient in case 1 had no evidence of significant stenosis as demonstrated in figures 8-9. His vasospastic event was related to his underlying pulmonary disorder and likely active history of smoking. He was started on isosorbide mononitrate $30 \mathrm{mg}$ daily, amlodipine $5 \mathrm{mg}$ daily and discharged home with close outpatient follow up. 


\section{Cureus}

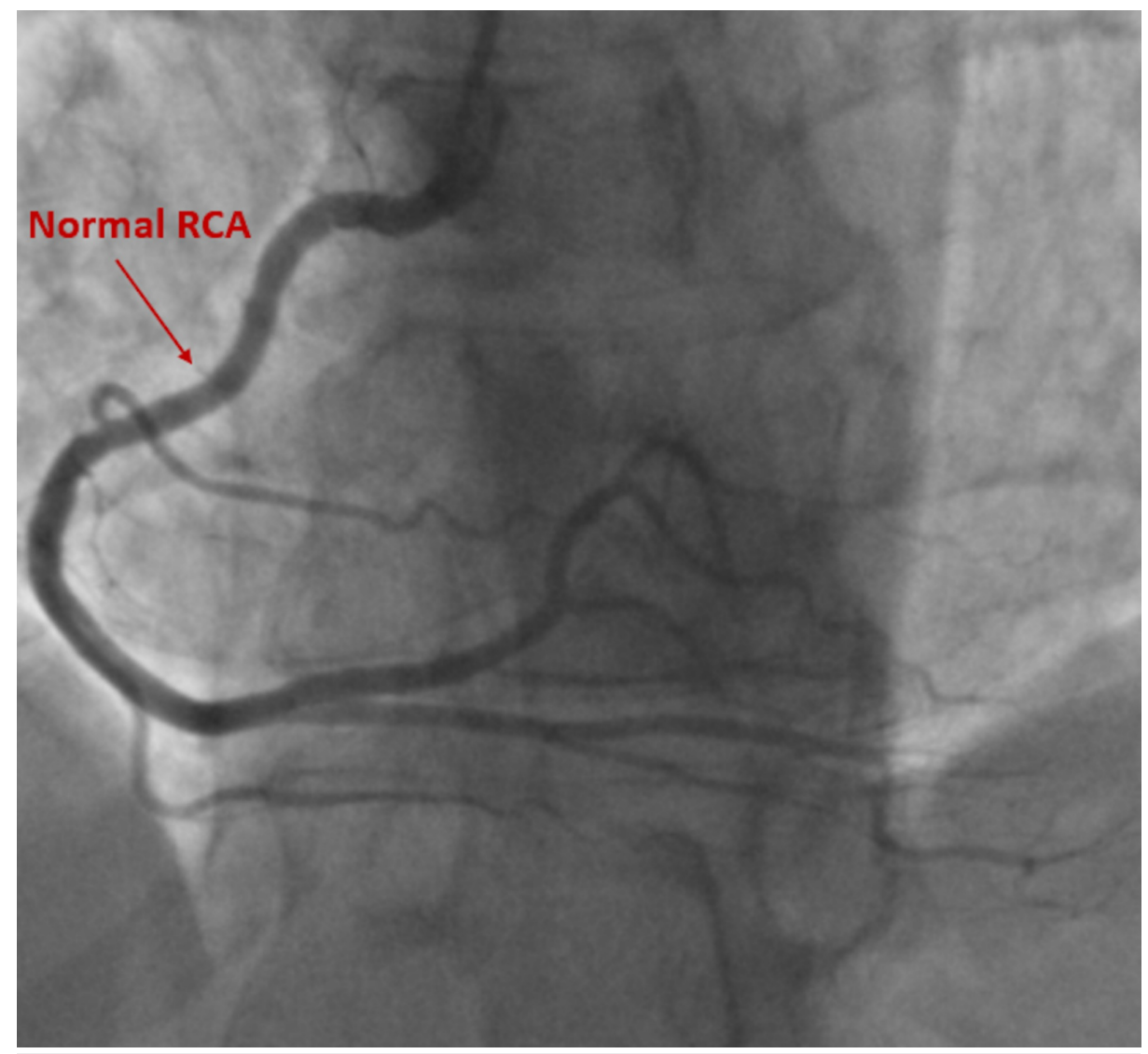

FIGURE 8: Cardiac catheterization with no evidence of disease within the right coronary artery

Right Coronary Artery (RCA)

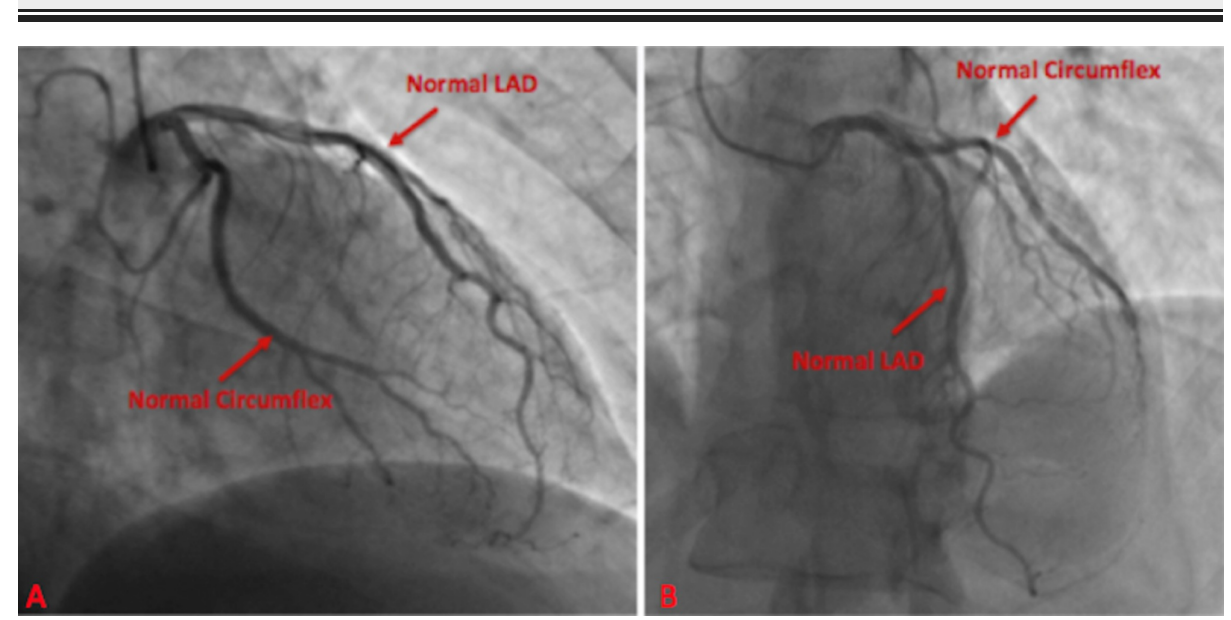

FIGURE 9: Cardiac catheterization at two viewing angles demonstrated negative disease in the left circumflex artery and left anterior descending artery

Left Anterior Descending (LAD)

The patient in case 2 demonstrated multi-vessel CAD with 70-80\% stenosis and 90-95\% stenosis in the mid right circumflex artery (RCA) (figure 10A), along with $70-80 \%$ stenosis in mid left circumflex artery (LCX) and $80-90 \%$ stenosis in distal LCX (figure 10B). The patient successfully underwent PCI with a drug eluding stent (DES) in the RCA and a stent in the LCX (figures 10C-10D). He was started on dual antiplatelet therapy 


\section{Cureus}

with daily aspirin $81 \mathrm{mg}$ daily and clopidogrel $75 \mathrm{mg}$ daily, along with atorvastatin $80 \mathrm{mg}$ daily for his underlying CAD. Patient was discharged home with long-acting nitrates and calcium channel blockade for prevention and treatment of vasospastic angina events.

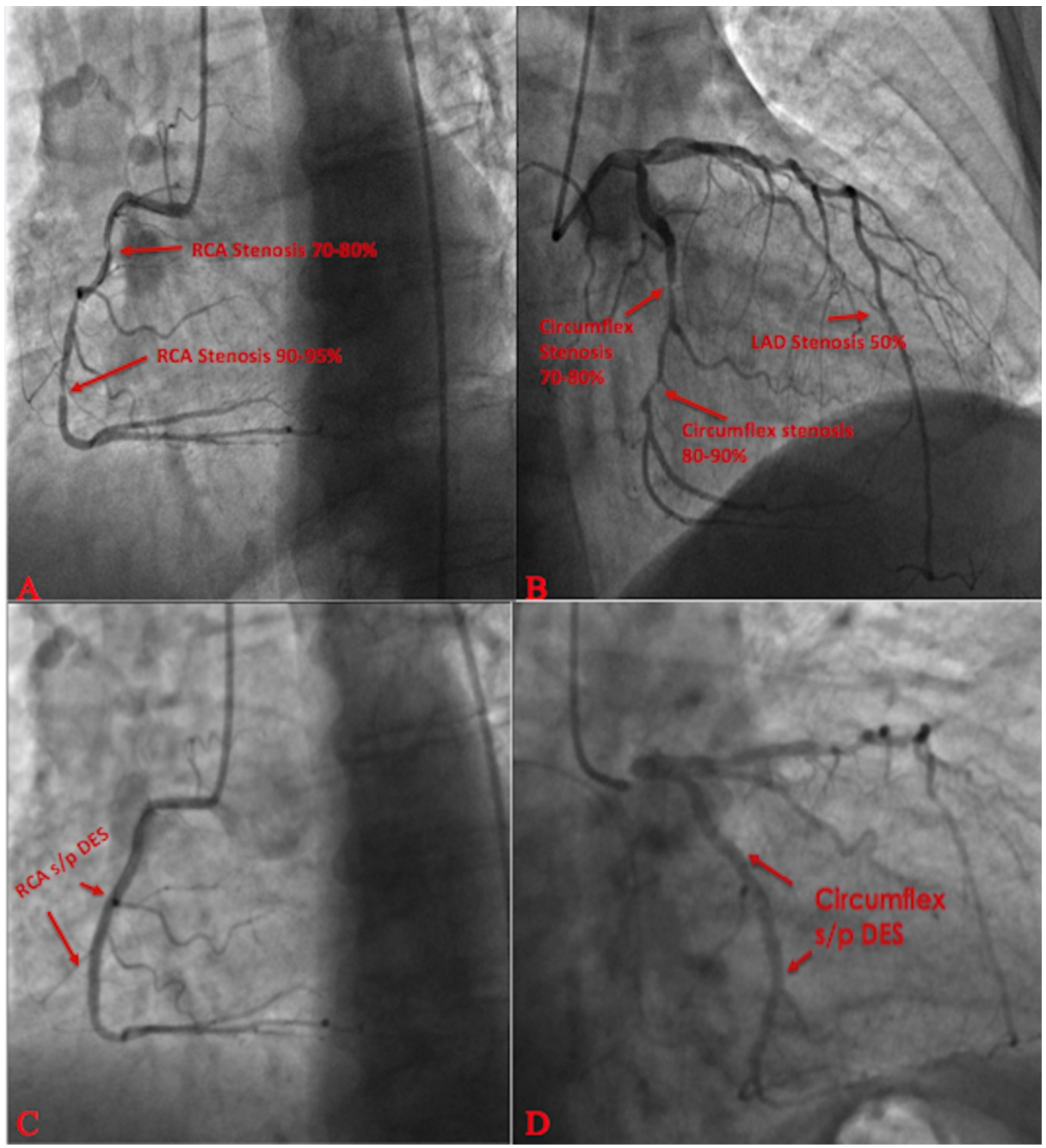

FIGURE 10: Left heart catheterization. a) Significant sequential stenotic lesions in mid right coronary artery b) Significant mid and distal left circumflex artery stenosis c) Right circumflex artery post drug eluding stent d) Left circumflex artery post drug eluding stent

Status post (S/P); Drug Eluding Stent (DES); Right Circumflex Artery (RCA); Left Anterior Descending (LAD)

\section{Discussion}

Although often a benign condition, vasospastic angina can cause malignant arrhythmias and cardiac ischemia. Causes range from tobacco use to coronary artery disease. It is important to rule out underlying $\mathrm{CAD}$ as the primary cause. In each case, both patients developed sudden onset substernal chest pain during their hospitalization with EKGs demonstrating ST elevations. Each patient received nitrates with resolution of their chest pain and resolution of ST elevations on subsequent EKGs, consistent with vasospastic angina. This demonstrates the importance of obtaining EKGs during the event and obtaining a repeat EKG after nitrate administration. Each patient had no previous history of CAD, but was taken for cardiac catheterization to rule out CAD as a primary cause. Patient in case 1 had no evidence of coronary disease. However, patient in case 2 was found to have severe multi-vessel disease with RCA and LCX stenosis requiring $\mathrm{PCI}$ with DES placement. CAD is a known cause of vasospastic angina, signifying the importance of diagnostic cardiac catheterization to rule significant cardiac disease. For patients with no evidence of significant CAD, other causes need to be excluded to prevent episodes from reoccurring. Patients who are smoking need to be counseled on importance of cessation. Regardless of underlying cause, treatment consists of long-acting nitrates and calcium channel blockers. First line treatment with calcium channel blockers and long-acting nitrates, in addition to smoking cessation, are class I indications according to 
American College of Cardiology [7]. Survival with medical therapy at five years can be as high as $94 \%$, however, those with underlying $\mathrm{CAD}$ have an overall worse prognosis.

\section{Conclusions}

Patients who experience an episode of vasospastic angina need to undergo further investigation in the form of a cardiac catheterization to rule out $\mathrm{CAD}$ as the underlying cause. It is important for clinicians to recognize vasospastic angina as this could precipitate fatal arrhythmias and cardiac ischemia resulting in increased mortality. Proper recognition, diagnosis and management can help decrease overall mortality in patients.

\section{Additional Information}

\section{Disclosures}

Human subjects: Consent was obtained by all participants in this study. Conflicts of interest: In compliance with the ICMJE uniform disclosure form, all authors declare the following: Payment/services info: All authors have declared that no financial support was received from any organization for the submitted work. Financial relationships: All authors have declared that they have no financial relationships at present or within the previous three years with any organizations that might have an interest in the submitted work. Other relationships: All authors have declared that there are no other relationships or activities that could appear to have influenced the submitted work.

\section{References}

1. Mann DL TG, Braunwald E: Braundwalds heart disease a textbook of cardiovascular medicine . Elsevier, Philadelphia; 2008.

2. Kaski JC, Crea F, Meran D, et al.: Local coronary supersensitivity to diverse vasoconstrictive stimuli in patients with variant angina. Circulation. 1986, 74:1255-1265. 10.1161/01.cir.74.6.1255

3. Kaski JC, Maseri A, Vejar M, et al.: Spontaneous coronary artery spasm in variant angina is caused by a local hyperreactivity to a generalized constrictor stimulus. J Am Coll Cardiol. 1989, 14:1456-1463. 10.1016/07351097(89)90382-3

4. Kusama Y, Kodani E, Nakagomi A, et al.: Variant angina and coronary artery spasm: the clinical spectrum, pathophysiology, and management. J Nippon Med Sch. 2011, 78:4-12. 10.1272/jnms.78.4

5. Ahn J-M, Lee KH, Yoo S-Y, et al.: Prognosis of variant angina manifesting as aborted sudden cardiac death . Journal of the American College of Cardiology. 2016, 68:137-145.

6. Beltrame JF, Crea F, Kaski JC, et al.: International standardization of diagnostic criteria for vasospastic angina. Eur Heart J. 2017, 38:2565-2568. 10.1093/eurheartj/ehv351

7. Al-Khatib SM, Stevenson WG, Ackerman MJ, et al.: 2017 AHA/ACC/HRS guideline for management of patients with ventricular arrhythmias and the prevention of sudden cardiac death: A report of the American College of Cardiology/American Heart Association task force on clinical practice guidelines and the Heart Rhythm Society. JACC. 2018, 72:91-220. 10.1016/j.jacc.2017.10.054 